\title{
Strong Shape Derivative for the Wave Equation with Neumann Boundary Condition
}

\author{
Jean-Paul Zolésio ${ }^{1}$ and Lorena Bociu ${ }^{2}$ \\ 1 CNRS-INLN, 1136 route des Lucioles, 06902 Sophia Antipolis France and CRM \\ (Applied Math lab.) Montréal, Canada \\ Jean-Paul.Zolesio@inln.cnrs.fr \\ 2 NC State University, Department of Mathematics, Raleigh, NC 27695, USA
}

\begin{abstract}
The paper provides shape derivative analysis for the wave equation with mixed boundary conditions on a moving domain $\Omega_{s}$ in the case of non smooth neumann boundary datum. The key ideas in the paper are (i) bypassing the classical sensitivity analysis of the state by using parameter differentiability of a functional expressed in the form of Min-Max of a convex-concave Lagrangian with saddle point, and (ii) using a new regularity result on the solution of the wave problem (where the Dirichlet condition on the fixed part of the boundary is essential) to analyze the strong derivative.
\end{abstract}

\section{Introduction}

The aim of this paper is to give a full analysis of the shape differentiability for the solution to the wave equation with mixed boundary conditions on a moving domain $\Omega_{s}$. The shape derivative investigation has been solved for the wave equation with homogeneous and non-homogeneous Dirichlet boundary conditions [1, 9, 13]. The novelty and difficulty of the paper are represented by the fact that the wave equation has non-homogeneous Neumann boundary condition on part of the boundary of its geometrical domain. Moreover, the Neumann datum $g$ is non-smooth, i.e. $g \in H^{-1 / 2}\left(\partial \Omega_{s}\right)$.

First, we prove existence of weak shape derivative by using parameter differentiability of a functional expressed in the form of Min-Max of a convex-concave Lagrangian with saddle point [3, 13. This completely bypasses the classical sensitivity analysis of the state (solution) [9]. A lot of problems in shape sensitivity analysis can be expressed as a Min Max of some Lagrangian dependent on the domain $\Omega$. Using a velocity field of deformations $V$ over $\Omega$ one can build a family of perturbations $\Omega_{s}, s \geq 0$, and then the tunics extends to situation when after some change of variable the sensitivity analysis reduces to the study of the differentiability of a Min Max Lagrangian functional with respect to the parameter $s$ for fixed velocity fields $V$ and domains $\Omega$.

Then we use a new regularity result for the solution to the wave problem to analyze the strong derivative via a brute force estimate on the differential quotient. In particular, we study the variational solution $y_{s}$ in the space

D. Hömberg and F. Tröltzsch (Eds.): CSMO 2011, IFIP AICT 391, pp. 445-460, 2013.

(C) IFIP International Federation for Information Processing 2013 
$W^{1, \infty}\left(0, \tau, L^{2}\left(\Omega_{s}\right)\right) \cap L^{\infty}\left(0, \tau, H_{*}^{1}\left(\Omega_{s}\right)=\left\{\phi \in H^{1}\left(\Omega_{s}\right), \phi=0\right.\right.$ on $\left.\left.S\right\}\right)$ by taking advantage of the Dirichlet condition on the fixed part of the boundary $S$ and we derive sharp estimate for $y_{s}$ at the boundary $\Gamma_{s}$ in terms of geometrical constants, in view of controlling the differential quotient's regularity that is necessary in the proof of our main result.

The new results obtained in this paper are: (i) existence of strong material and shape derivatives for the solution to the wave problem with mixed boundary conditions, and (ii) the new wave equation that the shape derivative solves.

The rest of the paper is organized as follows. In Section 2, we provide a preliminary result on existence and uniqueness of a Galerkin solution for the classical wave equation with variable coefficients. This result will be needed in the proof of our main theorem. In Section 3, we introduce the PDE model for the wave equation on a moving domain $\Omega_{s}$ and briefly recall the velocity method from shape optimization. In Section 4, we prove existence for the weak material derivatives. Finally, in Section 5 we complete the analysis by proving existence of strong material and shape derivatives, using the "extractor strategy" introduced in [5, 6] and Fourier transform techniques.

\section{Galerkin Solution for the Wave Equation}

Let $D \subset \mathbb{R}^{N}$ be fixed (potentially included in a $C^{2}$ manifold). Let $\Omega \subset D$ be an open bounded domain, with smooth boundary $\partial \Omega=\Gamma \cup S$, where $\bar{\Gamma} \cap \bar{S}=\emptyset$. Let $A(x)=\left\{a_{i j}(x)\right\}$ be a matrix of functions defined on $\Omega$ with the following properties:

$$
\left\{\begin{array}{l}
a_{i j} \in L^{\infty}(\Omega), \text { and } \\
\exists \alpha>0 \text { such that } \forall x \in \Omega, \forall \zeta=\left\{\zeta_{i}\right\} \in \mathbb{R}^{N}, a_{i j} \zeta_{i} \zeta_{j} \geq \alpha|\zeta|^{2} .
\end{array}\right.
$$

We associate with the matrix $A(x)$ the following operator

$$
A y \stackrel{\text { def }}{=}-\operatorname{div}(A(x) \nabla y) \text {. }
$$

Given $\tau>0$ and the interval $I=[0,2 \tau]$, consider the following wave equation problem:

$$
\begin{cases}y_{t t}+A \cdot y=f & \Omega \\ \frac{\partial y}{\partial n_{A}}=g & \Gamma \\ y=0 & S \\ y(0)=y_{0}, \quad y_{t}(0)=y_{1} & \end{cases}
$$

Galerkin solution for (2.2) in $L^{2}\left(I, H^{1}(\Omega)\right) \cap H^{1}\left(I, L^{2}(\Omega)\right)$.

Let $H_{*}^{1}(\Omega)=\left\{\phi \in H^{1}(\Omega), \phi=0\right.$ on $\left.S\right\}$ with norm $\|\phi\|^{2}=\int_{\Omega}|\nabla \phi|^{2} d x$. 
Proposition 2.1. Let $\left(y_{0}, y_{1}\right) \in H_{*}^{1}(\Omega) \times L^{2}(\Omega)$ and $f \in L^{2}(I \times \Omega)$ or $f \in$ $H(\Omega):=W^{1, \infty}\left(I, H^{-1}(\Omega)\right)$, and $g \in G(\Gamma):=W^{1,1}\left(I, H^{-1 / 2}(\Gamma)\right)$. Then the solution y to (2.2) verifies $y \in E(\Omega):=L^{\infty}\left(I, H_{*}^{1}(\Omega)\right) \cap W^{1, \infty}\left(I, L^{2}(\Omega)\right)$. Moreover when $f \in L^{2}(I \times \Omega)$ ), the trace $y_{\Gamma}$ of $y$ at the boundary $\Gamma$ verifies :

$$
y_{\Gamma} \in F(\Gamma):=H^{1 / 2}\left(0, \tau, L^{2}(\Gamma)\right) \cap H^{-1 / 2}\left(0, \tau, H^{1}(\Gamma)\right),
$$

and we have the following estimates: There exists constants $c, k$ such that

$$
\|y\|_{E(\Omega)}^{2}=\left\|y_{t}\right\|_{L^{\infty}\left(I, L^{2}(\Omega)\right)}^{2}+\|y\|_{L^{\infty}\left(I, H_{*}^{1}(\Omega)\right)}^{2} \leq c\|\|(f, g)\|\|^{2}
$$

and

$$
\|y\|_{F(\Gamma)}^{2}=\|y\|_{H^{1 / 2}\left(0, \tau, L^{2}(\Gamma)\right)}^{2}+\|y\|_{H^{-1 / 2}\left(0, \tau, H^{1}(\Gamma)\right)}^{2} \leq k\|\|(f, g)\|\|^{2},
$$

where

$$
\begin{gathered}
\left.\|||(f, g)\|\right|^{2}=\|g\|_{W^{1,1}\left(I, H^{-1 / 2}(\Gamma)\right)}^{2}+\|f\|^{2} \\
+\left|y_{0}\right|_{H_{*}^{1}(\Omega)}^{2}+\left|y_{1}\right|_{L^{2}(\Omega)}^{2}+\|f(0)\|_{H^{-1}(\Omega)}^{2}+\|g(0)\|_{H^{-1 / 2}(\Gamma)}^{2}
\end{gathered}
$$

and

$$
\|f\|^{2}=\|f\|_{W^{1,1}\left(I, H^{-1}(\Omega)\right)}^{2} \text { or }\|f\|_{L^{2}\left(I, L^{2}(\Omega)\right)}^{2} .
$$

\section{The Wave Equation on $\Omega_{s}$}

Let $D \subset \mathbb{R}^{N}$ be fixed (potentially included in a $C^{2}$ manifold). Let $\Omega \subset D$ be an open bounded domain, with smooth boundary $\partial \Omega=\Gamma \cup S$, where $\bar{\Gamma} \cap \bar{S}=\emptyset$.

The moving domain: For $s \in\left[0, s^{*}\right]$, let $V$ be a smooth vector field,

$$
V \in C^{0}\left(\left[0, s^{*}\left[, C^{1}\left(D, \mathbb{R}^{N}\right)\right) \text { with } V \cdot n=0 \text { on } \partial D \text {, and } V=0 \text { on } S .\right.\right.
$$

The flow transformation associated to $V$ is given by:

$$
T_{s}(V): \bar{D} \rightarrow \bar{D}, \text { such that } T_{s}(V)(S)=S .
$$

Using $T_{s}(V)$, we build the family of perturbed domains $\left\{\Omega_{s}\right\}_{s}$ as follows: $\Omega_{s}=$ $T_{s}(V)(\Omega)$ and $\partial \Omega_{s}=S \cup \Gamma_{s}$, where $\Gamma_{s}=T_{s}(V)(\Gamma)$. The normal component of the vector field $V(s)$ on the boundary $\Gamma_{s}$ (called the "normal speed") is denoted by $v(s)$, i.e. $v(s)=\left\langle V(s), n_{s}\right\rangle$, where $n_{s}=\nabla b_{\Omega_{s}}$ where $b(s)=b_{\Omega_{s}}$ stands for the oriented distance function to $\Omega_{s}$. From [4], we know that its shape derivative verifies

$$
b^{\prime}(s)=-V(s) \circ p_{s} \text { in a neighborhood of } \Gamma_{s},
$$

where $p_{s}$ is the projection map onto $\Gamma_{s}$. Most of the time, the normal speed appears in the calculus evaluated at 0 , hence we will use the following notation $v=v(0)$ for it. 
The PDE model for the wave problem. Let $\Omega_{s} \subset D$ be a moving domain with boundary $\partial \Omega_{s}=\Gamma_{s} \cup S$, where $\bar{\Gamma}_{s} \cap \bar{S}=\emptyset$, and $S$ is fixed with respect to the parameter $s$.

We consider the solution $y^{s}$ to the wave equation in the cylinder $] 0, \tau\left[\times \Omega_{s}\right.$, with homogeneous Dirichlet condition on the fixed part $S$ of the boundary, and verifying a non homogeneous Neumann condition on the moving part $\Gamma_{s}$ :

$$
\begin{cases}y_{t t}^{s}-\Delta y^{s}=0 & \Omega_{s} \\ y^{s}=0 & S \\ \frac{\partial}{\partial n_{s}} y_{s}=g(s) & \Gamma_{s}\end{cases}
$$

For each $s$ let the boundary datum $g$ have the following regularity $g_{s} \in$ $W^{1,1}\left(I, H^{-1 / 2}\left(\Gamma_{s}\right)\right)$. Then we consider the element $y_{s} \in H_{s}=L^{2}\left(I, H_{*}^{1}\left(\Omega_{s}\right)\right) \cap$ $H^{1}\left(I, L^{2}\left(\Omega_{s}\right)\right)$ solution to

$$
\forall \phi_{s} \in H_{s}, \int_{0}^{2 \tau} \int_{\Omega_{s}}\left(-\frac{\partial}{\partial t} y_{s} \frac{\partial}{\partial t} \phi_{s}+\nabla y_{s} \cdot \nabla \phi_{s}\right) d x d t=\int_{0}^{2 \tau} \int_{\Gamma_{s}} g_{s} \phi_{s} d \Gamma_{s} d t
$$

As mentioned before, the goal of this paper is threefold. We want to prove existence of the material derivative $\dot{y}(\Omega ; V)=\left.\frac{\partial}{\partial s}\left[y_{s} \circ T_{s}\right]\right|_{\{s=0\}}$ and of the shape derivative $y^{\prime}(\Omega ; V)=\dot{y}(\Omega ; V)-\nabla y \cdot V(0)$, and to render the new wave problem whose solution is the shape derivative $y^{\prime}(\Omega ; V)$. The first step consists in proving the existence of weak material derivative.

\section{Weak Material Derivatives}

To prove existence of material derivatives, we will take advantage of the regularity of solution for the linear wave equation and use the parameter differentiability for any functional expressed in form of a Min Max of a convex-concave Lagrangian with saddle points. The complete prove of MinMax parameter differentiability under saddle point was given in 2. The result in case of single unique saddle point ( which is easier) is given in [3] with application to PDE problem. Of course on formal view point such results was known by ingeeniers as a "necessary expression" ( assume it is differentiable then such is the expression. The difficult part being to prove the differentiability it self)

Let $R \in L^{2}\left(I, H^{-1}(\Omega)\right)$ and $q \in H^{1 / 2}\left(0, \tau, H^{-1}(\Gamma)\right)$. We consider the transported solution $y^{s}$ in the non perturbed geometry, that is $y^{s}=y_{s} \circ T_{s}(V)$, and we set

$$
j(s)=\int_{0}^{\tau}\left(\int_{\Omega} y^{s} R d x+\int_{\Gamma} y^{s} q d \Gamma\right) d t .
$$

Then we obtain that

$$
j(s)=\operatorname{MinMax}_{\{(\phi ; \psi) \in \times\}} \quad \mathcal{L}(s, \phi, \psi),
$$


where the Lagrangian $\mathcal{L}$ is given by

$$
\begin{gathered}
\mathcal{L}(s, \phi ; \psi)=\int_{0}^{\tau}\left(\int_{\Omega} \phi R d x+\int_{\Gamma} \phi q d \Gamma\right) d t \\
+\int_{0}^{\tau} \int_{\Omega}\left(-J(s) \frac{\partial \phi}{\partial t} \frac{\partial \psi}{\partial t}+\langle A(s) \nabla \phi, \nabla \psi\rangle\right) d x d t-\int_{0}^{\tau} \int_{\Gamma} \omega(s) g_{s} \circ T_{s}(V) \psi d \Gamma d t
\end{gathered}
$$

where $J(s)=\operatorname{det}\left(D T_{s}\right)$ is the Jacobian of the transformation $T_{s}(V)$,

$$
A(s)=J(s)\left(D T_{s}\right)^{-1}\left(D T_{s}\right)^{-*},
$$

and the density $\omega(s)$ is given as

$$
\omega(s)=\operatorname{det}\left(D T_{s}\right)\left|\left(D T_{s}(V)\right)^{-*} n_{s}\right| .
$$

At $s=0$ the unique saddle point of $\mathcal{L}$ is $(y, p)$, where the co-state $p$ is the solution to the following problem:

$$
\frac{\partial^{2}}{\partial t^{2}} p-\Delta p=R, \quad \frac{\partial}{\partial n} p=q .
$$

We derive that the functional $\mathcal{L}$ is differentiable and we get the explicit expression for its derivative w.r.t $s$ at $s=0$ :

$$
\begin{gathered}
j^{\prime}(0)=\frac{\partial}{\partial s} \mathcal{L}(0, y, p) \\
=\int_{0}^{\tau} \int_{\Omega}\left(-\operatorname{div} V(0) \frac{\partial y}{\partial t} \frac{\partial p}{\partial t}+\langle[\operatorname{div} V(0)-2 \epsilon(V(0))] \nabla y, \nabla p\rangle\right) d x d t \\
-\int_{0}^{\tau} \int_{\Gamma} p(\dot{g}(V)+H g v) d \Gamma d t
\end{gathered}
$$

where $2 \epsilon(V)=D V+(D V)^{*}, \dot{g}(V)=\left[\frac{d}{d s} g_{s} \circ T_{s}(V)\right]_{s=0}, v=\langle V(0), n\rangle$, and $H$ is the mean curvature of the boundary.

As a conclusion we get the existence of the weak derivative of the map $s \rightarrow y^{s}$ in $L^{2}\left(0, \tau, H^{1}(\Omega)\right)$, and the weak differentiability of the trace mapping: $\left.s \rightarrow y^{s}\right|_{\Gamma}$ in $H^{-1 / 2}\left(0, \tau, H^{1}(\Gamma)\right)$.

\section{$5 \quad$ Strong Material Derivative}

Theorem 5.1. Assume $g_{s} \in W^{1,1}\left(I, H^{-1 / 2}\left(\Gamma_{s}\right)\right)$ such that there exists $\dot{g} \in$ $L^{1}\left(I, H^{-1 / 2}(\Gamma)\right)$ verifying

$$
\frac{g_{s} \circ T_{s}(V)}{s}-\dot{g}(V) \rightarrow 0 \text { strongly in } L^{1}\left(I, H^{-1 / 2}(\Gamma)\right) \text {. }
$$

Then the solution $y_{s}$ has a strong material derivative in the following topology:

$\frac{y_{s} \circ T_{s}(V)-y}{s}-(\dot{Y}-\operatorname{div} V(0) y) \rightarrow 0$ in $L^{\infty}\left(0, \tau, L^{2}(\Omega)\right) \cap W^{-1, \infty}\left(0, \tau, H_{*}^{1}(\Omega)\right)$,

where $\dot{Y}$ is the solution to problem (5.10). 
Corollary 5.1.1. Let $g_{s} \in W^{2,1}\left(I, H^{-1 / 2}\left(\Gamma_{s}\right)\right)$ such that there exists $\dot{g} \in W^{1,1}\left(I, H^{-1 / 2}(\Gamma)\right)$ verifying

$$
\frac{g_{s} \circ T_{s}(V)}{s}-\dot{g}(V) \rightarrow 0 \text { strongly in } W^{1,1}\left(I, H^{-1 / 2}(\Gamma)\right) \text {. }
$$

Then the solution $y_{s}$ has a strong boundary material derivative in the following topology:

$$
\frac{y_{s} \circ T_{s}(V)-y}{s}-(\dot{Y}-\operatorname{div} V(0) y) \rightarrow 0 \text { in } L^{\infty}\left(0, \tau, H^{1 / 2}(\Gamma)\right),
$$

where $\dot{Y}$ is the solution to problem (5.10).

\subsection{Shape Derivative}

We know that

$$
\begin{array}{r}
\exists \mathcal{Y} \in C^{0}\left\{\left[0, s *\left[, W^{1, \infty}\left(I, L^{2}(D)\right) \cap L^{\infty}\left(I, H_{*}^{1}(D)\right)\right\} \cap C^{1}\left\{\left[0, s_{*}\left[, W^{1, \infty}\right.\right.\right.\right.\right. \\
\left.\left(I, H^{-1}(D)\right) \cap L^{\infty}\left(I, L^{2}(D)\right)\right\}
\end{array}
$$

such that

$$
\forall s, \quad \mathcal{Y}(s, .)=y_{s}(.) \text { on } \Omega_{s}
$$

Now the term

$$
y^{\prime}(\Omega ; V):=\left[\frac{\partial}{\partial s} \mathcal{Y}(0, x)\right]_{\{x \in \Omega\}} \in W^{1, \infty}\left(I, H^{-1}(\Omega)\right) \cap L^{\infty}\left(I, L^{2}(\Omega)\right)
$$

is independent of the choice of the function $\mathcal{Y}$ and is given by

$$
y^{\prime}(\Omega ; V)=\dot{y}(\Omega ; V)-\nabla y \cdot V(0) .
$$

For simplicity we write $\dot{y}$ and $y^{\prime}$ for the material and shape derivatives.

\subsubsection{Characterization of $y^{\prime}(\Omega ; V)$}

Proposition 5.1. The element $y^{\prime}$ is solution to the wave problem:

$$
\left\{\begin{array}{l}
\frac{\partial^{2}}{\partial t^{2}} y^{\prime}-\Delta y^{\prime}=0 \text { in } \Omega \\
\frac{\partial}{\partial n} y^{\prime}=\operatorname{div}_{\Gamma}\left(y \nabla_{\Gamma} v\right)-v \frac{\partial^{2} y}{\partial t^{2}}+H g v+g_{\Gamma}^{\prime} \text { on } \Gamma,
\end{array}\right.
$$

where $g_{\Gamma}^{\prime}$ stands for the boundary shape derivative of $g$ given by

$$
g_{\Gamma}^{\prime}=\dot{g}-\nabla_{\Gamma} g \cdot V_{\Gamma}(0) .
$$

The proof is done in several steps. Let $Y_{s}=\int_{0}^{t} y_{s}(\sigma) d \sigma$ and $G_{s}=\int_{0}^{t} g_{s}(\sigma) d \sigma$ be the solution to

$$
\forall \phi_{s} \in H(D), \int_{0}^{2 \tau} \int_{\Omega_{s}}\left(-\frac{\partial}{\partial t} Y_{s} \frac{\partial}{\partial t} \phi_{s}+\nabla Y_{s} . \nabla \phi_{s}\right) d x d t=\int_{0}^{2 \tau} \int_{\Gamma_{s}} G_{s} \phi_{s} d \Gamma_{s} d t .
$$


From 5.9 we get

$$
\begin{aligned}
\left\|\frac{\partial}{\partial t} Y_{s}\right\|_{L^{\infty}\left(I, L^{2}\left(\Omega_{s}\right)\right)}^{2}+\left\|Y_{s}\right\|_{L^{\infty}\left(I, H_{*}^{1}\left(\Omega_{s}\right)\right)}^{2} \leq & c\left(\left\|G_{s}\right\|_{L^{\infty}\left(I, H^{-1 / 2}\left(\Gamma_{s}\right)\right)}\right. \\
& \left.+\left\|g_{s}\right\|_{L^{1}\left(I, H^{-1 / 2}\left(\Gamma_{s}\right)\right)}\right)
\end{aligned}
$$

Consider the symmetrical matrix $A(s)=J(s) D T_{s}(V)^{-1} \cdot D T_{s}(V)^{-*} \in$ $L^{\infty}\left(D, R^{N^{2}}\right)$. Setting $Y^{s}=J_{s} Y_{s} \circ T_{s}(V)$ we get $\left(\right.$ with $\left.\phi_{s}=\psi \circ T_{s}(V)^{-1}\right)$

$$
\begin{aligned}
\forall \psi \in H=H_{0}, \int_{0}^{2 \tau} \int_{\Omega}\left(-\frac{\partial}{\partial t} Y^{s} \frac{\partial}{\partial t} \psi+\right. & A(s) \cdot \nabla\left(J_{s}^{-1} Y^{s}\right) \cdot \nabla \psi d x d t \\
& =\int_{0}^{2 \tau} \int_{\Gamma} G^{s} w(s) \psi d \Gamma d t
\end{aligned}
$$

Concerning the continuity of $s \rightarrow Y^{s}$ let $Z^{s}=Y^{s}-Y \in H^{1}\left(I, L^{2}(\Omega)\right) \cap$ $L^{2}\left(I, H_{*}^{1}(\Omega)\right)$, setting $\boldsymbol{m}(s)=\left(D T_{s}^{-1} \cdot D T_{s}^{-*}-I\right) \cdot \nabla_{\Gamma} Y^{s}+Y^{s} A \cdot \nabla\left(J_{s}^{-1}\right)+G^{s}$ $\left(D T_{s}^{-1} \cdot D T_{s}^{-*}-I\right) \cdot n$

Lemma 5.1. the term $\frac{1}{s}\|m(s)\|_{L^{\infty}\left(0,2 \tau, L^{2}(\Omega)\right)}$ remains bounded when $s \rightarrow 0$ Indeed from classical estimates ( see [9]) we have

$$
\left\|D T_{s}^{-1} \cdot D T_{s}^{-*}-I\right\|_{L^{\infty}(D)^{N^{2}}}+\left\|A(s) \cdot \nabla J_{s}^{-1}\right\|_{L^{\infty}(D)} \leq C_{V} s
$$

We get with 5.6

$$
\frac{1}{s}\|m(s)\|_{L^{\infty}\left(0,2 \tau, L^{2}(\Omega)\right)} \leq C_{V} c\left(\left\|G_{s}\right\|_{L^{\infty}\left(I, H^{-1 / 2}\left(\Gamma_{s}\right)\right)}+\left\|g_{s}\right\|_{L^{1}\left(I, H^{-1 / 2}\left(\Gamma_{s}\right)\right)}\right)
$$

and rom the sharp regularity at the boundary 2.4 we get

\section{Lemma 5.2.}

$$
m(s) \in H^{1 / 2}\left(0, \tau, L^{2}(\Gamma)\right)
$$

with the following estimate

$$
\begin{gathered}
\frac{1}{s}\|m(s)\|_{H^{1 / 2}\left(0, \tau, L^{2}(\Gamma)\right)}^{2} \leq c C_{V} \\
\left\|G^{s} w(s)-G\right\|_{L^{\infty}(\Gamma)} \leq C_{V} s
\end{gathered}
$$

The element $Z^{s}$ is solution of, $\forall \psi \in H=H_{0}$

$$
\begin{aligned}
\int_{0}^{2 \tau} \int_{\Omega}\left(-\frac{\partial}{\partial t} Z^{s} \frac{\partial}{\partial t} \psi+\right. & <\nabla Z^{s}, \nabla \psi>d x d t=-\int_{0}^{2 \tau} \int_{\Omega}<m(s), \nabla \psi>d x d t \\
& +\int_{0}^{2 \tau} \int_{\Gamma}\left(G^{s} w(s)-G\right) \psi d \Gamma d t
\end{aligned}
$$


That is

$$
\begin{aligned}
\int_{0}^{2 \tau} \int_{\Omega}\left(-\frac{\partial}{\partial t} Z^{s} \frac{\partial}{\partial t} \psi+\right. & <\nabla Z^{s}, \nabla \psi>d x d t=-\int_{0}^{2 \tau} \int_{\Omega} \bar{f}(s) \psi d x d t \\
& +\int_{0}^{2 \tau} \int_{\Gamma} \bar{g}(s) \psi d \Gamma d t
\end{aligned}
$$

Where

$$
\begin{gathered}
\bar{f}(s)=-\operatorname{div}(\boldsymbol{m}(s)) \rightarrow 0 \text { in } W^{1,1}\left(I, H^{-1}(\Omega)\right) \\
\bar{g}(s)=G^{s} \omega(s)-G+<\boldsymbol{m}(s), \boldsymbol{n}>\rightarrow 0 \text { in } W^{1,1}\left(I, H^{-1 / 2}(\Gamma)\right)
\end{gathered}
$$

From 2.5 we get:

$$
\begin{gathered}
\left\|Z_{t}\right\|_{L^{\infty}\left(I, L^{2}(\Omega)\right)}^{2}+\|Z\|_{L^{\infty}\left(I, H_{*}^{1}(\Omega)\right)}^{2} \leq c\left(\left\|G^{s} \omega-G+<m(s) . n>\right\|_{L^{\infty}\left(I, H^{-1 / 2}(\Gamma)\right)}\right. \\
\left.+\left\|g^{s} \omega-g+<\frac{\partial}{\partial t} m(s), n>\right\| \|_{L^{1}\left(I, H^{-1 / 2}(\Gamma)\right)}\right) \\
+\|\bar{f}\|_{L^{\infty}\left(I, H^{-1}(\Omega)\right)}+\left\|\frac{\partial}{\partial t} \bar{f}\right\|_{L^{1}\left(I, H^{-1}(\Omega)\right)}+\left[c\|\|^{2}+\|\| \|\right. \\
\left.2+\|\bar{f}(0)\|_{H^{-1}(\Omega)}+\|\bar{G}(0)\|_{H^{-1 / 2}(\Gamma)}\right]^{1 / 2}
\end{gathered}
$$

Proposition 5.2. Assume that $s \rightarrow g^{s}$ is continuous in $L^{1}\left(I, H^{-1 / 2}(\Gamma)\right)$.

We consider the element $\dot{Y} \in H_{0}$ solution to the problem

$$
\begin{gathered}
\forall \psi \in H=H_{0}, \int_{0}^{2 \tau} \int_{\Omega}\left(-\frac{\partial}{\partial t} \dot{Y} \frac{\partial}{\partial t} \psi+\nabla \dot{Y} \cdot \nabla \psi\right) d x d t \\
=\int_{0}^{2 \tau} \int_{\Omega}<\nabla(Y \operatorname{div} V(0))-\dot{A} \cdot \nabla Y, \nabla \psi>d x d t \\
\quad+\int_{0}^{2 \tau} \int_{\Gamma}(\dot{G}+G H v) \psi d \Gamma d t \\
=\int_{0}^{2 \tau}<\operatorname{div}([\dot{A}-\operatorname{div} V(0) I] . \nabla Y), \psi>_{H_{\bar{\Omega}}^{-1}(D) \times H^{1}(\Omega)} d t \\
+\int_{0}^{2 \tau} \int_{\Gamma}(\dot{G}+G H-<\dot{A} \cdot n, \nabla Y>+<\nabla(Y \operatorname{div} V(0)), n>) \psi d \Gamma d t
\end{gathered}
$$

This problem is relevant from the previous variational approach as the right hand side

$$
\begin{gathered}
f=\operatorname{div}(\dot{A} \nabla Y-\nabla Y \operatorname{div} V(0))=-2 \operatorname{div}(\epsilon(V(0)) . \nabla Y) \in H^{1}\left(I, H^{-1}(\Omega)\right) \\
\bar{g}=-<\dot{A} \cdot n, \nabla Y>+<\nabla(Y \operatorname{div} V(0)), n> \\
=2<\epsilon(V) . \nabla Y, n>+Y \frac{\partial}{\partial n} \operatorname{div} V(0) \in H^{1 / 2}\left(I, L^{2}(\Gamma)\right)
\end{gathered}
$$




\subsection{Differential Quotient}

We consider the elements

$$
d(s)=\frac{y^{s}-y}{s}-\dot{y}, \quad \delta(s)=\frac{Y^{s}-Y}{s}-\dot{Y}
$$

In order to characterise $\delta(s)$ we introduce the following vectors functions:

$$
\begin{gathered}
\mathcal{M}_{1}(s)=\left[\frac{D T_{s}^{-1} \cdot D T_{s}^{-*}}{s}-2 \epsilon V(0)\right] \cdot \nabla Y^{s} \\
\mathcal{M}_{2}(s)=2 \epsilon(V) \cdot \nabla\left(Y^{s}-Y\right) \\
\mathcal{M}_{3}(s)=\left[Y^{s} A \cdot\left(\frac{\nabla\left(J_{s}^{-1}\right)}{s}-\nabla \operatorname{div} V(0)\right)+\left(Y I-Y^{s} A\right) \cdot \nabla \operatorname{div} V(0)\right] \\
\mathcal{M}(s)=\mathcal{M}_{1}(s)+\mathcal{M}_{2}(s)+\mathcal{M}_{3}(s)
\end{gathered}
$$

And the function on the boundary:

$$
G_{\delta}(s)=\frac{G^{s} \omega(s)-G}{s}-(\dot{G}+G H v)
$$

the element $\delta(s)$ is then solution to

$$
\begin{aligned}
\forall \psi, \int_{0}^{2 \tau} \int_{\Omega}\left(-\frac{\partial}{\partial t} \delta \frac{\partial}{\partial t} \psi+\nabla \delta . \nabla \psi\right) d x d t=- & \int_{0}^{2 \tau} \int_{\Omega}<\mathcal{M}, \nabla \psi>d t \\
& +\int_{0}^{2 \tau} \int_{\Gamma} G_{\delta}(s) \psi d \Gamma d t
\end{aligned}
$$

That is

$$
\begin{gathered}
\int_{0}^{2 \tau} \int_{\Omega}\left(-\frac{\partial}{\partial t} \delta \frac{\partial}{\partial t} \psi+\nabla \delta . \nabla \psi\right) d x d t= \\
\int_{0}^{2 \tau}<f_{\delta}(s), \psi>_{H_{\bar{\Omega}}^{-1}(D) \times H^{1}(\Omega)} d t+\int_{0}^{2 \tau} \int_{\Gamma} g_{\delta}(s) \psi d \Gamma d t
\end{gathered}
$$

Where

$$
\begin{gathered}
f_{\delta}(s)=\operatorname{div}(\mathcal{M}) \rightarrow 0 \text { in } H^{1}\left(I, H^{-1}(\Omega)\right), s \rightarrow 0, \\
g_{\delta}(s)=G_{\delta}(s)-\mathcal{M} . n \rightarrow 0 \text { in } H^{1}\left(I, H^{-1 / 2}(\Gamma)\right), s \rightarrow 0 .
\end{gathered}
$$

\subsection{Fourier Transform}

From now on we assume the data, $y_{0}=y_{1}=0$ and the Neumann data $g \in$ $W^{1,1}\left(I, H^{-1 / 2}(\Gamma)\right)$ being approached in this space by a smooth element $g^{m}$. Then the associated solution $y^{m}$ is smoother. We consider a smooth cuting function $0 \leq \rho(t) \leq 1$ such that $\rho(t)=0$ for $|t| \geq 2 \tau$ while $\rho(t)=1$ when $|t| \leq \tau$. We consider $\left(y^{m}\right)^{0},\left(g^{m}\right)^{0}$ the extension by zero out of $I$ and we set

$$
\tilde{y}^{m}(t, x)=\rho(t)\left(y^{m}\right)^{0}(t, x), \quad \tilde{g}^{m}(t, x)=\rho(t)\left(g^{m}\right)^{0}(t, x)
$$


With

$$
F^{m}=\rho f+2 \rho_{t} y_{t}^{m}+\rho_{t t} y^{m} \rightarrow F=\rho f+2 \rho_{t} y_{t}+\rho_{t t} y \text { in } L^{2}\left(I, L^{2}(\Omega)\right) \text { as } m \rightarrow \infty .
$$

We get

$$
\tilde{y}_{t t}^{m}+A . \tilde{y}^{m}=F^{m}, \frac{\partial \tilde{y}^{m}}{\partial n_{A}}=\tilde{g}^{m} \text {, on } \Gamma, \tilde{y}^{m}=0 \text { on } S .
$$

For each $m$ we consider the Fourier transform

$$
\begin{array}{r}
\mathcal{F}^{m}(\zeta, x)=\int_{-\infty}^{+\infty} \tilde{F}^{m}(t, x) e^{-i \zeta t} d t, z^{m}(\zeta, x)=\int_{-\infty}^{+\infty} \tilde{y}^{m}(t, x) e^{-i \zeta t} d t \\
\mathcal{G}^{m}(\zeta, x)=\int_{-\infty}^{+\infty} \tilde{g}^{m}(t, x) e^{-i \zeta t} d t \\
-\zeta^{2} z^{m}+A . z^{m}=\mathcal{F}^{m}, \quad \frac{\partial z^{m}}{\partial n_{A}}=\mathcal{G}^{m}, \text { on } \Gamma, z^{m}=0 \text { on } S
\end{array}
$$

5.3.1 Extractor. Given $\mu>0$, consider the velocity field $V \in C^{0}\left(\left[0, \mu\left[; W^{2, \infty}\right.\right.\right.$ $\left(D, \mathbf{R}^{N}\right)$ ) and its associated flow mapping $T_{s}(V)$. Given $s \geq 0$, denote by $\Omega_{s}=T_{s}(V)(\Omega)$ the perturbed domain with boundary $\Gamma_{s}=T_{s}(V)(\Gamma)$. Consider the functional

$$
\begin{aligned}
\mathcal{E}^{m}(s, V) \stackrel{\text { def }}{=} & \int_{-\infty}^{+\infty} d \zeta \int_{\Omega_{s}(V)}\left[|\zeta|\left|z^{m} \circ T_{s}(V)^{-1}\right|^{2}\right. \\
& +\frac{1}{1+|\zeta|} \mid<A . \nabla\left(z^{m} \circ T_{s}(V)^{-1}\right) \\
& \left.\left.\nabla\left(z^{m} \circ T_{s}(V)^{-1}\right)>\mid\right)\right] d x
\end{aligned}
$$

and its derivative

$$
\left.e^{m \stackrel{\text { def }}{=}} \frac{d}{d s} \mathcal{E}^{m}(s, V)\right|_{s=0}
$$

that will be computed in two different ways. For simplicity in the following computations we denote by $V$ the autonomeous vector field $V(0)$. Derivative by moving boundary results : Let

$$
\begin{aligned}
e_{1}^{m} \stackrel{\text { def }}{=} \int_{-\infty}^{+\infty} d \zeta \int_{\Omega}|\zeta| 2 \mathcal{R} e\left\{z^{m} \nabla z^{\bar{m}} \cdot(-V)\right\} & \\
& +\frac{1}{1+|\zeta|} 2 \mathcal{R} e\left\{<A \cdot \nabla\left(z^{m}\right), \nabla\left(\nabla z^{\bar{m}}(-V)>\right)\right\} d x \\
& +\int_{-\infty}^{+\infty} d \zeta\left(\int_{\Gamma}\left\{|\zeta|\left|z^{m}\right|^{2}+\frac{1}{1+|\zeta|}<A . \nabla z^{m}, \nabla z^{m}>\right\}<V, n>d \Gamma(x)\right) .
\end{aligned}
$$


Consider the first integral term over $\Omega$ :

$$
\begin{array}{r}
a \stackrel{\text { def }}{=} \int_{-\infty}^{+\infty} d \zeta \int_{\Omega} \\
\left(|\zeta| 2 \mathcal{R} e\left\{z^{m} \nabla z^{\bar{m}} \cdot(-V)\right\}-\frac{1}{1+|\zeta|} 2 \mathcal{R} e\left\{<A . \nabla z^{m}, \nabla\left(\nabla z^{\bar{m}} \cdot V\right)>\right\}\right) d x
\end{array}
$$

By Stokes theorem using the fact that $\partial z^{m} / \partial n_{A}=\mathcal{G}^{m}$, we get the following expression:

$$
\begin{aligned}
a= & \int_{-\infty}^{+\infty} d \zeta \int_{\Omega}\left(|\zeta| 2 \mathcal{R} e\left\{-z^{m} \nabla z^{\bar{m}} \cdot V\right\}+\frac{1}{1+|\zeta|} 2 \mathcal{R} e\left\{\operatorname{div}\left(A \cdot \nabla z^{m}\right) \nabla z^{\bar{m}} \cdot V\right\}\right) d x \\
& -\int_{-\infty}^{+\infty} d \zeta \int_{\Gamma} \frac{1}{1+|\zeta|} 2 \mathcal{R} e\left\{\mathcal{G}^{m} \nabla z^{\bar{m}} \cdot V\right\} d \Gamma
\end{aligned}
$$

As we have $-\operatorname{div}\left(A . \nabla z^{m}\right)=A \cdot z^{m}=\left(\zeta^{2} z^{m}+\mathcal{F}^{m}(\zeta, x)\right)$ we get

$$
\begin{aligned}
a= & 2 \mathcal{R} e\left\{\int_{-\infty}^{+\infty} d \zeta \int_{\Omega}\left(-z^{m}\left(|\zeta|+\frac{\zeta^{2}}{1+|\zeta|}\right)\right) \nabla z^{\bar{m}} \cdot V d x\right\} \\
& +2 \mathcal{R} e\left\{\int_{-\infty}^{+\infty} d \zeta \int_{\Omega} \frac{1}{1+|\zeta|} \mathcal{F}^{m} \nabla z^{\bar{m}} \cdot V d x\right\} \\
& -\int_{-\infty}^{+\infty} d \zeta \int_{\Gamma} \frac{1}{1+|\zeta|} 2 \mathcal{R} e\left\{\mathcal{G}^{m} \nabla z^{\bar{m}} \cdot V\right\} d \Gamma
\end{aligned}
$$

Lemma 5.3. For each $m>0$ we get

$$
\begin{aligned}
e_{1}^{m}= & \int_{-\infty}^{+\infty} d \zeta\left(\int_{\Gamma}\left\{|\zeta||z|^{2}+\frac{1}{1+|\zeta|}<A \cdot \nabla z^{m}, \nabla z^{m}>\right\}<V, n>d \Gamma(x)\right) \\
& -\int_{-\infty}^{+\infty} d \zeta \int_{\Gamma} \frac{1}{1+|\zeta|} 2 \mathcal{R} e\left\{\mathcal{G}^{m} \nabla z^{\bar{m}} \cdot V\right\} d \Gamma \\
& +2 \mathcal{R} e\left\{\int_{-\infty}^{+\infty} d \zeta \int_{\Omega}\left(-z^{m}\left(|\zeta|+\frac{\zeta^{2}}{1+|\zeta|}\right) \nabla z^{\bar{m}} \cdot V d x\right\}\right. \\
& +2 \mathcal{R} e\left\{\int_{-\infty}^{+\infty} d \zeta \int_{\Omega} \frac{1}{1+|\zeta|} \mathcal{F}^{m} \nabla z^{\bar{m}} \cdot V d x\right\} .
\end{aligned}
$$

5.3.2 Derivative by change of variable $T_{s}(V)$ Consider now the expression

$$
\mathcal{E}^{m}(s, V) \stackrel{\text { def }}{=} \int_{-\infty}^{+\infty} d \zeta \int_{\Omega}\left[|\zeta|\left|z^{m}\right|^{2}+\frac{1}{1+|\zeta|}<B(s) . \nabla z^{m}, \nabla z^{m}>\right] j(s) d x
$$

where

$$
B(s) \stackrel{\text { def }}{=} D T_{s}(V)^{-1} . A \circ T_{s}(V) .\left(D T_{s}(V)^{-1}\right)^{*}, \quad j(s) \stackrel{\text { def }}{=} \operatorname{det} D T_{s}(V)
$$


and

$$
\left(B^{\prime} \cdot V\right)=-D V \cdot A-A \cdot D V^{*}+\nabla A \cdot V
$$

where $\nabla A . V$ is the matrix

$$
(\nabla A . V)_{i, j} \stackrel{\text { def }}{=} \nabla a_{i, j} . V .
$$

Then we get

\section{Lemma 5.4.}

$$
\begin{aligned}
e_{2}^{m}= & \int_{-\infty}^{+\infty} d \zeta \int_{\Omega}\left[|\zeta|\left|z^{m}\right|^{2}+\frac{1}{1+|\zeta|}<A \cdot \nabla z^{m}, \nabla z^{m}>\right] \operatorname{div} V d x \\
& +\int_{-\infty}^{+\infty} d \zeta \int_{\Omega} \frac{1}{1+|\zeta|}<\left(B^{\prime} \cdot V\right) \cdot \nabla z^{m}, \nabla z^{m}>d x
\end{aligned}
$$

\subsection{Extractor Identity}

We now equate the two expressions, $e_{1}^{m}=e_{2}^{m}$, to get

\section{Lemma 5.5.}

$$
\begin{aligned}
& \int_{-\infty}^{+\infty} d \zeta\left(\int_{\Gamma}\left\{|\zeta|\left|z^{m}\right|^{2}+\frac{1}{1+|\zeta|}<A \cdot \nabla z^{m}, \nabla z^{m}>\right\}<V, n>d \Gamma(x)\right) \\
& -\int_{-\infty}^{+\infty} d \zeta \int_{\Gamma} \frac{1}{1+|\zeta|} 2 \mathcal{R} e\left\{\mathcal{G}^{m} \nabla z^{\bar{m}} \cdot V\right\} d \Gamma \\
= & -2 \mathcal{R} e\left\{\int_{-\infty}^{+\infty} d \zeta \int_{\Omega}\left(-z^{m}\left(|\zeta|+\frac{\zeta^{2}}{1+|\zeta|}\right)\right) \nabla z^{\bar{m}} \cdot V d x\right\} . \\
& -2 \mathcal{R} e\left\{\int_{-\infty}^{+\infty} d \zeta \int_{\Omega} \frac{1}{1+|\zeta|} \mathcal{F}^{m} \nabla z^{\bar{m}} \cdot V d x\right\} \\
& +\int_{-\infty}^{+\infty} d \zeta \int_{\Omega}\left[|\zeta|\left|z^{m}\right|^{2}+\frac{1}{1+|\zeta|}<A \cdot \nabla z^{m}, \nabla z^{m}>\right] \operatorname{div} V d x \\
& +\int_{-\infty}^{+\infty} d \zeta \int_{\Omega} \frac{1}{1+|\zeta|}<\left(B^{\prime} \cdot V\right) \cdot \nabla z^{m}, \nabla z^{m}>d x .
\end{aligned}
$$

The velocity vector $V$ will be choosen in terms of the geometry of the boundary which is best handled by using the oriented distance function, (cf. [7]) $b_{\Omega}=$ $d_{\Omega}-d_{\Omega^{c}}, \Omega^{c}=\mathbf{R}^{N} \backslash \Omega$. When $\Omega$ is of class $C^{1,1}$, the unit outward normal $n$ to the boundary $\Gamma=\partial \Omega$ is equal to $\nabla b_{\Omega}$. Moreover when $\Gamma$ is compact, there exist $h>0$ and an $h$-tubular neighboorhood $\mathcal{U}_{h}=\left\{x \in \mathbf{R}^{N}:\left|b_{\Omega}(x)\right|<h\right\}$ of $\Gamma$ such that $b_{\Omega} \in C^{1,1}\left(\mathcal{U}_{h}\right)$. In order to work in $\mathbf{R}^{\mathrm{N}}$, localize the oriented distance function to this neighboorhood and work with a global $C^{1,1}$ function on $\mathbf{R}^{N}$, define $b_{\Omega}^{h} \stackrel{\text { def }}{=} \theta^{h} \circ b_{\Omega}$ for some function $\theta^{h} \in C^{1,1}(\mathbf{R} ;[0,1])$ such that

$$
\theta^{h}(t) \stackrel{\text { def }}{=} \begin{cases}1, & |t|<h / 3 \\ 0, & |t|>2 h / 3 .\end{cases}
$$


Obviously $b_{\Omega}^{h}$ is equal to $b_{\Omega}$ in $\mathcal{U}_{h / 3}$ and $\operatorname{supp} b_{\Omega}^{h} \subset \mathcal{U}_{h}$, with $b_{\Omega}^{h} \in C^{1,1}(\overline{\mathbf{R}})$ with the unit outward normal $n=\nabla b_{\Omega}=\nabla b_{\Omega}^{h}$ on $\Gamma$. In order to take care of the boundary condition term $\mathcal{G}$ in the extractor identity we have to make a specific choice for the vector field $V$. Choose $V$ in the following form (here we assume the matrix $A$ to be defined in the neighbourhood of $\bar{\Omega}): V \stackrel{\text { def }}{=} A . \nabla b_{\Omega}^{h} \Rightarrow V=$ A.n on $\Gamma$, so that $\nabla z^{m} . V=<\nabla z^{m}, A . n>=\mathcal{G}^{m}$, while $v=<V, n>=<A$. $n, n>$ $\geq \alpha>0$ on $\Gamma$. So the first term yields

$$
\begin{aligned}
& \alpha \int_{-\infty}^{+\infty} d \zeta\left(\int_{\Gamma}\left\{|\zeta|\left|z^{m}\right|^{2}+\frac{1}{1+|\zeta|}\left|\nabla z^{m}\right|^{2}\right\} d \Gamma(x)\right) \\
& \leq \int_{-\infty}^{+\infty} d \zeta \int_{\Gamma} \frac{1}{1+|\zeta|} 2 \mathcal{R} e\left\{\mathcal{G}^{m} \overline{\mathcal{G}}^{m}\right\} d \Gamma \ldots \\
& \quad . \mid-2 \mathcal{R} e\left\{\int_{-\infty}^{+\infty} d \zeta \int_{\Omega}\left(-z^{m}\left(|\zeta|+\frac{\zeta^{2}}{1+|\zeta|}\right)\right) \nabla z^{m} \cdot V d x\right\} . \\
& \quad-2 \mathcal{R} e\left\{\int_{-\infty}^{+\infty} d \zeta \int_{\Omega} \frac{1}{1+|\zeta|} \mathcal{F}^{m} \nabla z^{m} \cdot V d x\right\} \\
& \quad+\int_{-\infty}^{+\infty} d \zeta \int_{\Omega}\left[|\zeta|\left|z^{m}\right|^{2}+\frac{1}{1+|\zeta|}<A . \nabla z^{m}, \nabla z^{m}>\right] \operatorname{div} V d x \\
& \quad+\int_{-\infty}^{+\infty} d \zeta \int_{\Omega} \frac{1}{1+|\zeta|}<\left(B^{\prime} \cdot V\right) \cdot \nabla z^{m}, \nabla z^{m}>d x \mid .
\end{aligned}
$$

and we obtain the following estimate.

Proposition 5.3. There exists a constant $M>0$ such that for all $m>0$

$$
\begin{aligned}
\int_{-\infty}^{+\infty} d \zeta & \left(\int_{\Gamma}\left\{|\zeta|\left|z^{m}\right|^{2}+\frac{1}{1+|\zeta|}\left|\nabla z^{m}\right|^{2}\right\} d \Gamma(x)\right) \\
\leq \frac{1}{\alpha} M\{ & \left\|\sqrt{\frac{1}{1+|\zeta|}} \mathcal{G}^{m}\right\|_{L^{2}\left(R_{\zeta}, L^{2}(\Gamma)\right)}^{2} \\
& +\|V(0)\|_{L^{\infty}\left(D, \mathbf{R}^{N}\right)}\left\|z^{m}\right\|_{L^{2}\left(\mathbf{R}_{\zeta}, L^{2}(\Omega)\right)}\left\|\nabla z^{m}\right\|_{L^{2}\left(\mathbf{R}_{\zeta}, L^{2}\left(\Omega, \mathbf{R}^{N}\right)\right)} \\
& +\|V(0)\|_{L^{\infty}\left(D, \mathbf{R}^{N}\right)}\left\|\sqrt{\frac{1}{1+|\zeta|}} \mathcal{F}^{m}\right\|_{L^{2}\left(\mathbf{R}_{\zeta}, L^{2}(\Omega)\right)}\left\|\nabla z^{m}\right\|_{L^{2}\left(\mathbf{R}_{\zeta}, L^{2}\left(\Omega, \mathbf{R}^{N}\right)\right)} \\
& \left.+\left\|\sqrt{\zeta} z^{m}\right\|_{L^{2}\left(\mathbf{R}_{\zeta}, L^{2}(\Omega)\right)}\|\operatorname{div} V(0)\|_{L^{\infty}(D)}\left\|\left(B^{\prime} . V\right)+A\right\|_{L^{\infty}\left(D, \mathbf{R}^{N}\right)}\right\} \\
& +\left\|\sqrt{\frac{1}{1+|\zeta|}} \nabla z^{m}\right\|_{L^{2}\left(\mathbf{R}_{\zeta}, L^{2}\left(\Omega, \mathbf{R}^{N}\right)\right)}
\end{aligned}
$$

As $V=A . \nabla b_{\Omega}^{h}$, we get

$$
\|\operatorname{div} V(0)\|_{L^{\infty}(D)} \leq M_{3}\left(\|A\|_{W^{1, \infty}(\Omega)}+\left\|\Delta b_{\Omega}^{h}\right\|_{L^{\infty}(D)}\right)
$$


while

$$
\left\|B^{\prime} . V\right\|_{L^{\infty}(D)} \leq M_{4}\left(\|A\|_{W^{1, \infty}(\Omega)}+\left\|D^{2} b_{\Omega}^{h}\right\|_{L^{\infty}(D)}\|A\|_{L^{\infty}\left(D, \mathbf{R} N^{2}\right)}\right)
$$

Each terms depending on $m$ in the right-hand side of (5.16) converges in the respective norms:

$$
\mathcal{G}^{m} \rightarrow \mathcal{G}, \quad \mathcal{F}^{m} \rightarrow \mathcal{F}, \quad z^{m} \rightarrow z
$$

So that there exists a constant $M_{2}>0$ such that, for all $m>0$ we have:

$$
\forall m>0, \quad \int_{-\infty}^{+\infty} d \zeta\left(\int_{\Gamma}\left\{|\zeta|\left|z^{m}\right|^{2}+\frac{1}{1+|\zeta|}\left|\nabla z^{m}\right|^{2}\right\} d \Gamma(x)\right) \leq M_{2} .
$$

Consider the following two measures on $\mathbf{R}_{\zeta}$ :

$$
\mu_{1}(\zeta) \stackrel{\text { def }}{=} \sqrt{|\zeta|} d \zeta \text { and } \mu_{2}(\zeta) \stackrel{\text { def }}{=} \sqrt{\frac{1}{1+|\zeta|}} d \zeta
$$

Then we get

$$
\left\|z^{m}\right\|_{L_{\mu_{1}}^{2}\left(\mathbf{R}_{\zeta}, L^{2}(\Gamma)\right)} \leq M_{2} \quad \text { and } \quad\left\|\nabla z^{m}\right\|_{L_{\mu_{2}}^{2}\left(\mathbf{R}_{\zeta}, L^{2}\left(\Gamma, \mathbf{R}^{N}\right)\right)} \leq M_{2}
$$

and there exist elements $\phi, \boldsymbol{\Phi}$ such that for the two associated weighted topologies we get the weak convergences:

$$
\begin{gathered}
z_{\Sigma}^{m} \rightarrow \phi \text { weakly in } L_{\mu_{1}}^{2}\left(\mathbf{R}_{\zeta}, L^{2}(\Gamma)\right) \\
\nabla z_{\Sigma}^{m} \rightarrow \Phi \text { weakly in } L_{\mu_{2}}^{2}\left(\mathbf{R}_{\zeta}, L^{2}\left(\Gamma, \mathbf{R}^{N}\right)\right)
\end{gathered}
$$

Obviously $\phi=\left.z\right|_{\sigma}$ and $\Phi=\left.\nabla \phi\right|_{\Sigma}$ then $\Phi=\left.\nabla z\right|_{\Sigma}$ and the norms being weakly l.s.c. in the limit we get the estimate:

$$
\int_{-\infty}^{+\infty} d \zeta\left(\int_{\Gamma}\left\{|\zeta||z|^{2}+\frac{1}{1+|\zeta|}|\nabla z|^{2}\right\} d \Gamma(x)\right) \leq M_{2}
$$

By Plancherel isomorphism we get in the real line, we get

\section{Corollary 5.1.2.}

$$
\begin{aligned}
& |\zeta|^{1 / 2} z \in L^{2}\left(\mathbf{R}, L^{2}(\Gamma)\right) \Longleftrightarrow \tilde{y} \in H^{1 / 2}\left(\mathbf{R}, L^{2}(\Gamma)\right), \\
& \left((1+|\zeta|)^{-1 / 2}\right) \nabla z \in L^{2}\left(\mathbf{R}, L^{2}\left(\Gamma, \mathbf{R}^{N}\right)\right) . \quad \Longleftrightarrow \nabla \tilde{y} \in H^{-1 / 2}\left(\mathbf{R}, L^{2}\left(\Gamma, \mathbf{R}^{N}\right)\right) \text {. }
\end{aligned}
$$

Obviously we have:

$$
\|y\|_{H^{1 / 2}\left(I, L^{2}(\Gamma)\right)} \leq\|\tilde{y}\|_{H^{1 / 2}\left(\mathbf{R}, L^{2}(\Gamma)\right)}
$$

Also:

$$
\left.\nabla y\right|_{\Sigma}=\nabla_{\Gamma} y+\frac{\partial y}{\partial n} n
$$


But

$$
\frac{\partial y}{\partial n}=g \in L^{2}(\Sigma)
$$

So that

$$
\nabla_{\Gamma} y \in H^{-1 / 2}\left(\mathbf{R}, L^{2}\left(\Gamma, \mathbf{R}^{N}\right)\right)
$$

Which implies

$$
\left.y\right|_{\Sigma} \in H^{-1 / 2}\left(\mathbf{R}, H^{1}(\Gamma)\right)
$$

from which, as $y \in C^{0}\left([0, \tau], H^{3 / 5-\sigma}(\Omega)\right)$, we get (??).

Proposition 5.4. Let $\Gamma$ be a $C^{2}$ submanifold in $\mathbf{R}^{N}$ and $g \in L^{2}\left(I ; L^{2}(\Gamma)\right)$, $f \in L^{2}\left(I ; L^{2}(\Omega)\right), y_{0} \in H^{2}(\Omega), y_{1} \in L^{2}(\Omega)$, and $A \in W^{1, \infty}\left(D, \mathbf{R}^{N^{2}}\right)$. Then the trace at the boundary $\Gamma$ of $y$ verifies the regularity (2.4).

We have:

$\|y\|_{H^{1 / 2}\left(0, \tau, L^{2}(\Gamma)\right)}^{2}+\|y\|_{H^{-1 / 2}\left(0, \tau, H^{1}(\Gamma)\right)}^{2} \leq\|\tilde{y}\|_{H^{1 / 2}\left(0,2 \tau, L^{2}(\Gamma)\right)}^{2}+\|\tilde{y}\|_{H^{-1 / 2}\left(0,2 \tau, H^{1}(\Gamma)\right)}^{2}$

from the Plancherel isometry in the estimation 5.16, we get:

$$
\begin{gathered}
\leq C\left\{\|\tilde{g}\|_{H^{-1 / 2}\left(0,2 \tau, L^{2}(\Gamma)\right)}^{2}+\|\tilde{y}\|_{H^{-1 / 2}\left(0,2 \tau, H^{1}(\Omega)\right)}^{2}+\|\tilde{F}\|_{H^{-1 / 2}\left(0,2 \tau, L^{2}(\Omega)\right)}^{2}\right. \\
+\|\tilde{y}\|_{H^{1 / 2}\left(0,2 \tau, L^{2}(\Omega)\right)}^{2}+\|\nabla \tilde{y}\|_{H^{-1 / 2}\left(0,2 \tau, L^{2}(\Omega)\right)}^{2}
\end{gathered}
$$

and obviously

$$
\begin{gathered}
\leq C_{2}\left\{\|g\|_{H^{1 / 2}\left(0,2 \tau, L^{2}(\Gamma)\right)}^{2}+\|y\|_{H^{-1 / 2}\left(0,2 \tau, H^{1}(\Omega)\right)}^{2}+\|F\|_{H^{-1 / 2}\left(0,2 \tau, L^{2}(\Omega)\right)}^{2}\right. \\
+\|y\|_{H^{1 / 2}\left(0,2 \tau, L^{2}(\Omega)\right)}^{2}+\|\nabla y\|_{H^{-1 / 2}\left(0,2 \tau, L^{2}(\Omega)^{N}\right)}^{2}
\end{gathered}
$$

5.4.1 Boundedness of $\boldsymbol{F}$. From 5.12 there exists a constant $c_{\rho}>0$ such that

$$
\begin{aligned}
\|F\|_{H^{-1 / 2}\left(0,2 \tau, L^{2}(\Omega)\right)} \leq\|F\|_{L^{2}\left(0,2 \tau, L^{2}(\Omega)\right)} \leq c_{\rho}\left\{\left\|y_{t}\right\|_{L^{2}(I \times \Omega)}^{2}\right. & +\|y\|_{L^{2}(I \times \Omega)}^{2} \\
& \left.+\|f\|_{L^{2}(I \times \Omega)}^{2}\right\}
\end{aligned}
$$

and from 5.9 we get

$$
\begin{array}{r}
\|F\|_{H^{-1 / 2}\left(0,2 \tau, L^{2}(\Omega)\right)} \leq\|F\|_{L^{2}\left(0,2 \tau, L^{2}(\Omega)\right)} \leq c\left(\left\|\left.f\right|_{L^{2}(I \times \Omega)}+\right\| g(t) \|_{L^{\infty}\left(I, H^{-1 / 2}(\Gamma)\right)}\right. \\
\left.+\left\|\frac{\partial}{\partial t} g(t)\right\|_{L^{1}\left(I, H^{-1 / 2}(\Gamma)\right)}\right) \\
+\|f\|_{L^{\infty}\left(I, H^{-1}(\Omega)\right)}+\left\|\frac{\partial}{\partial t} f\right\|_{L^{1}\left(I, H^{-1}(\Omega)\right)} \\
+\left[c E(0)+\|f(0)\|_{H^{-1}(\Omega)}\right. \\
\left.+\|g(0)\|_{H^{-1 / 2}(\Gamma)}\right]^{1 / 2}
\end{array}
$$


Theorem 5.2. Let $g \in W^{1,1}\left(0,2 \tau, H^{-1 / 2}(\Gamma)\right) \cap H^{1 / 2}\left(0,2 \tau, L^{2}(\Gamma)\right), f \in L^{2}(I \times$ $\Omega) \cap W^{1,1}\left(I, H^{-1}(\Omega)\right)$. Then there exist a constant $k$ depending on the domain $\Omega$, on the $L^{\infty}\left(I, W^{1, \infty}(\Omega)\right)$-norm of the coefficients matrix $A$, on the cutting function $\rho$ and on the trace of the linear trace operator (restriction to $\Gamma$ in the norm of $\mathcal{L}\left(H_{*}^{1}(\Omega), H^{1 / 2}(\Gamma)\right)$ such that:

$$
\begin{aligned}
\|y\|_{H^{1 / 2}\left(0, \tau, L^{2}(\Gamma)\right)}^{2}+\|y\|_{H^{-1 / 2}\left(0, \tau, H^{1}(\Gamma)\right)}^{2} \leq k\left\{\left(\|\left. f\right|_{L^{2}(I \times \Omega)}\right.\right. & \\
& \left.+\|g(t)\|_{L^{\infty}\left(I, H^{-1 / 2}(\Gamma)\right)}+\left\|\frac{\partial}{\partial t} g(t)\right\|_{L^{1}\left(I, H^{-1 / 2}(\Gamma)\right)}\right) \\
& +\|f\|_{L^{\infty}\left(I, H^{-1}(\Omega)\right)}+\left\|\frac{\partial}{\partial t} f\right\|_{L^{1}\left(I, H^{-1}(\Omega)\right)} \\
& \left.+\left[E(0)+\|f(0)\|_{H^{-1}(\Omega)}+\|g(0)\|_{H^{-1 / 2}(\Gamma)}\right]^{1 / 2}\right\}
\end{aligned}
$$

\section{References}

[1] Cagnol, J., Zolésio, J.-P.: Shape derivative in the wave equation with Dirichlet boundary conditions. J. Differential Equations 158(2), 175-210 (1999)

[2] Correa, R., Seeger, A.: Directional derivative of an minmax function. Nonlinear Anal. 9, 13-22 (1985)

[3] Cuer, M., Zolésio, J.-P.: Control of singular problem via differentiation of a minmax. Systems \& Control Letters 11(2), 151-158 (1988)

[4] Delfour, M., Zolésio, J.-P.: Shapes and Geometries. Analysis, Differential Calculus, and Optimization. SIAM Advances in Design and Control (2001)

[5] Delfour, M.C., Zolésio, J.-P.: Hidden boundary smoothness for some classes of differential equations on submanifolds, Optimization methods in partial differential equations. Contemp. Math., vol. 209, pp. 59-73. Amer. Math. Soc. Providence, RI (1997)

[6] Delfour, M.C., Zolésio, J.-P.: Curvatures and skeletons in shape optimization. Z. Angew. Math. Mech. 76(3), 198-203 (1996)

[7] Desaint, F.R., Zolésio, J.-P.: Manifold derivative in the Laplace-Beltrami equation. J. Funct. Anal. 151(1), 234-269 (1997)

[8] Lasiecka, I., Triggiani, R.: Control theory for partial differential equation Encyclopedia of mathematics. Cambridge University Press (2000)

[9] Sokolowski, J., Zolésio, J.-P.: Introduction to shape optimization. Shape sensitivity analysis. Springer Ser. Comput. Math., vol. 16. Springer, Berlin (1992)

[10] Zolésio, J.-P.: The material derivative (or speed) method for shape optimization. In: Haug, E.J., Céa, J. (eds.) Optimization of Distributed Parameter Structures (Iowa City, Iowa, 1980). NATO Adv. Sci. Inst. Ser. E: Appl. Sci., vol. II, 50, pp. 1089-1151. Sijhofff and Nordhoff, Alphen aan den Rijn, Nijhoff, The Hague (1981)

[11] Zolésio, J.-P., Goatin, P.: N Dimensional Crowd Motion (in this book)

[12] Zolésio, J.-P.: Identification de Domaine, These de doctorat d'état, Nice, France (1979)

[13] Zolésio, J.-P.: Hidden boundary shape derivative for the solution to Maxwell equations and non cylindrical wave equations. In: Optimal Control of Coupled Systems of Partial Differential Equations. Internat. Ser. Numer. Math., vol. 158, pp. 319 345. Birkhuser Verlag, Basel (2009) 